\title{
Análisis psicoanalítico sobre las problemáticas en la identificación con la función parental en la adolescencia: la transición hacia la adultez como un espacio de transformación
}

\section{Psychoanalytic analysis about identification problems with the parental function in adolescence: transition toward adulthood} as a transformative space

Mediante una viñeta clínica se propone analizar cómo se lleva a cabo, durante la adolescencia, un proceso de identificación y transmisión de la función parental en la relación entre el adolescente y sus padres, que podría permitir pensar los avatares de los procesos de transición hacia la adultez. Se discute acerca de cómo la inclusión de estos procesos, en la clínica con adolescentes, podría enriquecer los análisis con perspectivas históricas e intersubjetivas que posibilitarian la reflexión sobre el rol de los padres en la clínica con adolescentes, el duelo respecto a la propia adolescencia y las problemáticas actuales en la transición hacia la adultez en adolescentes.

Palabras clave: Función parental, adolescencia, adultez, identificación

${ }^{* 1}$ Universidad Viña del Mar (Viña del Mar, Chile). 


\section{Introducción}

La clínica con adolescentes ha ido paulatinamente inscribiendo un campo propio de teoría y práctica, desprendiéndose de la práctica con niños para construir su particular trabajo de resignificación y reelaboración de las demandas pulsionales de la pubertad, con el fin de habilitar un proyecto identificatorio posible en su transición hacia la adultez. La pregunta en torno a la clínica con adolescentes en nuestro contexto epocal cobra sentido, debido a que con el paso de los años teóricamente se ha indagado en torno a la necesidad de pensar una práctica particular en el trabajo con adolescentes, desligándolo del trabajo infantil, o como se denomina "infanto juvenil", y con un contexto particular. El psicoanálisis refiere que pensar la adolescencia en nuestras condiciones contextuales implica la imposibilidad de una categorización unificante.

Así como la adolescencia y su conceptualización en la clínica - debido a su relación intrínseca con los procesos socioculturales y simbólicos - va variando y reestructurándose, ocurre algo similar a los padres y su lugar en la clínica psicoanalítica con adolescentes: "[...] las estructuras familiares se han modificado con un estancamiento de la familia nuclear (...)" (Valdés, 2007, p. 2). En el contexto clínico es posible observar las tensiones y movimientos que se reactualizan en el vínculo que los padres deben construir en este proceso, dentro del cual quedan a la deriva respecto a la intensidad de su participación, con el fin de no estar muy partícipes y por ende coartar los espacios de autonomía del adolescente, pero tampoco marginarse y dejar al adolescente como único actor del proceso terapéutico.

Autores como François Marty (2001, 2005, 2007; 2009a), François Richard (2009) y Phillipe Jeammet (1995), refieren la importancia de "hacer generación en la adolescencia" mediante la identificación a la función parental, es decir, "la capacidad que el 


\section{ARTIGOS}

adolescente adquiere para proyectarse como padre potencial, en el sentido de devenir responsable de sus actos y de sus pensamientos, el adolescente entra en la perspectiva de las generaciones" (Marty, 2009a, p. 77). Tales procesos psíquicos favorecen la distinción entre las generaciones y la marca o límite que organiza el espacio simbólico, permitiendo referirse como sujeto separado, y bajo esto advenir como adulto.

La transición hacia la adultez para adolescentes es un proceso que no es claro en los tiempos actuales. Si bien Erikson (1968) refería la "moratoria psicosocial" como periodo de exploración identitaria en la adolescencia, es relevante analizar qué factores psíquicos y contextuales posibilitan el abandono de la adolescencia. En general, la transición hacia la adultez ha sido mayormente abordada desde sus aspectos sociales (Marzana et al., 2012), siendo relevante poder analizar cómo los aspectos psíquicos, como la transmisión de la función parental, tienen lugar dentro de este proceso. La aproximación a esta temática podría facilitar la comprensión no sólo de la transición hacia la adultez en el contexto actual, sino a su vez de los elementos clínicos que pueden integrarse en el dispositivo terapéutico con jóvenes que se encuentran en esta transición.

Una manera de interpretar la perspectiva de las generaciones es a través de la relevancia de la función parental en la adolescencia, y, sobre todo, de su transmisión. La identificación en la adolescencia surge como un proceso secundario en el cual se subsanarían las angustias depresivas que surgen a partir de la separación necesaria para la construcción de la subjetividad adolescente. Mannoni (1984) describe la función de la identificación en la adolescencia como una problemática importante para la teoría psicoanalítica:

La oscuridad de los fenómenos de identificación es lo que hace difícil una teoría psicoanalítica de la adolescencia. El sujeto está obligado ¿Cómo? ¿Por qué? - a condenar las identificaciones pasadas. Sabe que ya no es un niño - y si no lo sabe no faltará quien se lo recuerde- pero sabe también que no es un adulto (algo que se le recuerda aun más) y que se expone al ridículo (que produce precisamente una ruptura de identificación en el nivel del yo), si se deja ir y cree que es un adulto". (p. 26)

Para Marty (2010), las identificaciones también son la base de la subjetivación, un proceso en la medida que permite la ocurrencia del despliegue de lo subjetivo, pero que si tienen una posible claridad respecto a su funcionamiento: "Las identificaciones son la base de los procesos de subjetivación en la medida que permitan su ocurrencia, de ahí la necesidad de observar sus métodos para tratar de evaluar la implementación de los obstáculos en este 
proceso, y en caso de fallas, para detectar la posibilidad de una reparación" (p. 191). El proceso de subjetivación significaría "hacer subjetivo" o "convertirse en sujeto", lo cual implica una recuperación que fuerza el movimiento identificatorio (Cahn, 1997).

El autor describe cómo el concepto de identificación resume el trabajo psíquico que debe enfrentar cualquier adolescente, principalmente frente dos tareas interrelacionadas: la integración de una nueva identidad sexual y autónoma en relación con sus objetos de amor que implica alteraciones internas y externas, conscientes e inconscientes que tiene como objetivo el cambiar sin dejar de ser uno mismo, tarea que fue descrita con anterioridad a partir de las conceptualizaciones psicoanalíticas de la adolescencia, en el sentido del cambio o muda de estructuración corporal y psíquica (Piccini, Barrionuevo y Vega, 2007). Este proceso es psíquico y no meramente cronológico, bajo lo cual no necesariamente sigue lineamientos normativos, siendo relevante pensar la particularidad de este proceso en la transición hacia la adultez.

La identificación con la función parental permite de este modo que el adolescente logre elaborar su propia fragilidad y agresividad. A partir de la identificación con la función que ejercen los padres, el adolescente puede 764 acceder a una operación identificatoria: "Ir más allá de los parental es acceder a este registro de identificación que no concierne a la persona de los padres (esta operación identificatoria ya se ha comprometido en la infancia), pero si a su función" (Marty, 2009a, p. 78).

La función parental refiere a la capacidad que el adolescente adquiere para proyectarse como padre potencial, en el sentido de devenir responsable de sus actos y de sus pensamientos. A través de esta identificación, el adolescente entra en la perspectiva de las generaciones: "él hace límite (a la infancia en él, como un punto de no retorno; también límite entre la infancia y la edad adulta) haciendo generación, se distingue activamente de la generación de sus padres y se proyecta en un porvenir donde él puede vivirse como un padre potencial" (Marty, 2009a, p. 77).

La relevancia de la identificación con la función parental apela a la noción de hacer generación, que no sólo implica la operación de separación y diferenciación referida anteriormente, sino que también la posibilidad de vivenciar el haber devenido uno mismo, alguien que pueda estar al origen de la vida y que pueda, a su vez, continuar la senda de sus propios padres: "Hacer generación, es hacer vivir en sí los proyectos, es imaginar crear acontecimientos, objetos (culturales), es sentirse a la medida para dar vida a otro diferente de s'́" (Marty, 2009a, p. 77). 


\section{ARTIGOS}

En base a estos antecedentes, se expondrá el análisis de una viñeta clínica en donde se busca ilustrar las dimensiones en las cuales la función parental se transmite en el trabajo clínico con adolescentes en su transición hacia la adultez. Si bien se recurre a una viñeta para discutir en torno a las problemáticas vinculadas a la identificación con la función parental en la transición hacia la adultez, el objetivo no es generalizar, sino ilustrar y evocar al lector respecto a casos similares, otorgando nuevos elementos de análisis en el dispositivo clínico con adolescentes en su transición hacia la adultez.

\section{El caso de Elizabeth: “¿Y qué pasa si me quedo sola?”}

Elizabeth tiene 18 años. Vive con su madre y padre, y su hermano menor de 9 años. Llega a la consulta con su madre debido a que, a juicio de la madre, "está muy cambiada, siempre callada, no nos cuenta nada y a veces la pillamos 1lorando". Elizabeth refiere que "no tiene nada que contar" y por eso prefiere guardar silencio. Para mantener la confidencialidad y aspectos éticos, nombres y datos fueron modificados.

En la primera entrevista con los padres y la adolescente, Elizabeth es descrita por la madre como una adolescente que prefiere estar en su pieza "encerrada y sola", sin compartir con sus padres y su hermano. Asiste a su último año de colegio, en donde tiene un grupo de amigas con quienes dice compartir gustos musicales y de canales de Youtube sobre maquillaje y música. Describe a su vez mantener relaciones de amistad con otras jóvenes de manera virtual, lo que su madre no aprueba debido a que lo considera como "peligroso".

Sus padres trabajan cerca de su hogar, lo cual facilita sus traslados. Para la madre esto es importante debido a que "no le gusta que Elizabeth o su hermano queden solos". La relación entre la pareja es descrita como estable, idea que es apoyada por Elizabeth.

De manera posterior, se realiza una entrevista solo con los padres de Elizabeth, quienes describen mucha preocupación sobre su hija, ya que sienten que ha existido un alejamiento respecto a ellos. Adicionalmente, exponen que han descubierto a Elizabeth llorar sola en su pieza, y cuando le han preguntado los motivos, ella describe que es por nada o que no sabe por qué. Ambos padres intentan construir teorías de los motivos de estos episodios, vinculándolo a elementos escolares ("quizás no le gusta el colegio") o problemas con sus amistades ("puede que pelee con sus amigas"). 
Durante las entrevistas con Elizabeth, inicialmente se muestra reacia a hablar debido a que "no le pasa nada". Con el paso del tiempo, y a través de incluir en sesión el relato de algunos de sus intereses como por ejemplo los canales de Youtube que suele observar, comienza a dar cuenta de sus motivaciones y sobre todo preocupaciones. Entre ellas emerge con recurrencia, a nivel de discurso y en producciones gráficas, la idea de la soledad como una instancia angustiante que relaciona con el "crecer": "Me da miedo ser grande" o "A veces veo en los videos que la vida de persona adulta es entretenida, pero no sé si yo lo haga bien". En una de las entrevistas preliminares describe: "Me da miedo estar sola, ¿qué pasa si me quedo sola?".

Al interpretar en conjunto sus dibujos y relatos, Elizabeth expone una preocupación importante respecto al miedo de estar sola, lo cual ella relaciona con el ser adulto. Esto hace que en ocasiones "se descontrole", como ella llama a sus ataques de llanto. Además, describe preocupación en elegir la carrera universitaria correcta al salir del colegio. A pesar de que debido a la edad de Elizabeth (18 años) se le considera legalmente como mayor de edad, se decide incluir de igual manera a los padres a través de la realización de entrevistas, en donde se indaga en torno a la habilitación de Elizabeth en

766 tanto sujeto adulto dentro del marco familiar, lo cual ellos describen como dificultoso porque siempre la han visto como "una niña nerviosa", lo cual es trabajado junto a los padres en sesiones sin la participación de Elizabeth.

En base a esto, se analizan tres ejes temáticos que pueden ayudar a discutir y comprender el proceso de transición hacia la adultez y sus dificultades: el problema del duelo de y en la adolescencia, la configuración de las identificaciones durante la adolescencia y la transmisión de la función parental.

\section{Análisis}

\section{a) El duelo de la adolescencia y el duelo en la adolescencia}

Dentro de la viñeta, y como ha sido discutido teóricamente (Marty, 2009a, b, 2010a, b; Gutton, 2006; Rother de Hornstein, 2006; Ferté-Marriaux, 2009; Horenstein, 2013), las relaciones familiares son de suma importancia en la adolescencia no sólo para el sujeto adolescente, sino también para quienes ejercen la función parental, en quienes también emergen distintas elaboraciones y configuraciones particulares frente a la adolescencia de su hijo. Uno de los 


\section{ARTIGOS}

trabajos más importantes para el adolescente es la separación de los padres, descrita como una de las tareas más difíciles que el sujeto debe enfrentar en su vida, pero al mismo tiempo la más necesaria (Freud, 1909/1992).

Esta separación no es sólo física, sino que implica procesos psíquicos particulares que pueden plantearse metapsicológicamente desde la noción de duelo (Freud, 1917[1915]/2013); Blos, 1976; Aberasruty y Knobel, 1998; Rother de Hornstein, 2006). Este proceso psíquico en torno a la pérdida ha sido conceptualizado teóricamente como el duelo de la infancia, en el sentido de la elaboración de la pérdida de la infancia. Sin embargo, en base a lo descrito en el caso, es posible postular la idea de un duelo de la propia adolescencia, en tanto proceso metapsicológico de abandono de este momento psíquico antes de advenir adulto en el contexto epocal.

La adolescencia pone en juego no sólo el duelo de la niñez en tanto apropiación del cuerpo púber y elaboración psíquica de la pérdida de la identidad infantil o las figuras parentales, sino a su vez, de manera posterior, un proceso de duelo respecto al abandono de la adolescencia y los procesos psíquicos de transformación relacionados. Autores como Cahn (1997) exponen cómo los procesos psíquicos adolescentes implican una similitud con los procesos limítrofes, bajo lo cual su permanencia sería patológica. En esta lógica puede pensarse que las características descritas como identidad inestable, reedición edipica y fragilidad narcisista (Le Breton, 2012) deben pasar a ser abandonadas en una cultura que favorece a su vez estas características (Untoiglich, 2009). Dentro del discurso de Elizabeth se expone cómo la adolescencia no es sólo una etapa cronológica, sino sobre todo un proceso psíquico y subjetivo (Cornejo, 2015), que implicaría por si mismo un proceso de duelo para advenir en tanto adulto.

La literatura ha expuesto que uno de los modos en que el adolescente hace frente al duelo respecto a la niñez, y sobre todo a la separación de las figuras parentales, es el grupo de pares, que a su vez propicia el proceso de duelo en los padres respecto a su hijo o hija adolescente. Es destacable cómo a la luz de la viñeta es posible problematizar la relevancia que lo virtual adquiere en este proceso de construcción de la alteridad en la adolescencia, considerando que para Elizabeth sus amigas virtuales eran más "importantes" que sus amigas del colegio, con quienes comparte el día a día. Esto, ya que lo virtual ofrecería una atemporalidad y una modalidad de cercanía-lejanía que, en este caso, es facilitadora de la intimidad: "Me siento más en confianza porque les puedo contar todo en cualquier momento y sin que me vean si me da vergüenza", expresa Elizabeth. 
Siguiendo lo propuesto por Freud (1917[1915]/2013), a través del duelo la pérdida del objeto se hace menos angustiante, ya que mediante las identificaciones que se hacen hacia el objeto de amor perdido se mantiene a nivel psíquico los aspectos queridos de este objeto. De este modo, se ha documentado cómo en el duelo en la adolescencia es importante la elaboración de la pérdida de las figuras parentales; pero, en el duelo de la adolescencia, la operación de elaborar la pérdida de la adolescencia en tanto etapa de transición posibilitaría el adviniendo subjetivo a partir de la separación no de los padres como figura, sino de las transmisiones psíquicas de los padres: "Un individuo joven sale de la adolescencia cuando la angustia de sus padres no le produce ningún efecto inhibidor" (Dolto, 1988, p. 21).

Gutton (1993, 2006) propone la idea de la "obsolescencia parental" en tanto proceso por el cual los padres deben advenir como sujetos desidealizados para el adolescente, idea que también puede ser interpretada a partir de los trabajos de Winnicott (1979). La diferenciación respecto a las figuras parentales como eje del desarrollo psíquico adolescente puede ser interpretado a su vez como una reorganización respecto a los discursos que han constituido la niñez del sujeto adolescente y que a su vez pasan a ser re-visitados durante la adolescencia. Esto genera un conflicto entre las generaciones, lo cual es propuesto por Winnicott $(1964 ; 1979)$ en tanto el pasaje se complica ya que el niño para hacerse adulto debe pasar sobre el cuerpo del adulto muerto simbólicamente. Este proceso es parte de la transición hacia la adultez, bajo lo cual existiría a su vez una operación psíquica en los padres respecto a su abandono como objetos idealizados. Este reconocimiento de separación frente a los discursos parentales, así como de posicionamiento de los padres dentro de la obsolescencia, posibilitaría el duelo de la adolescencia bajo la identificación con la función parental.

En el caso de Elizabeth, a modo de ilustración, surge una especie de inhibición del conflicto tal y como se ha descrito en los estudios psicoanalíticos sobre adolescencia, vinculada a la idea de rebelión y empuje pulsional puesta en acto (Winnicott, 1979; Rassial, 1999; Feixa, 2006; Le Breton, 2012; Klein, 2014; Bilbao y Jofré, 2017). En la actualidad parece emerger la noción de un conflicto en un plano virtual, mediado por los modos de lazo social en los cuales la adolescencia emerge. Es decir, el conflicto es la falta de comunicación o introversión (por ejemplo "estar encerrada en la pieza"), más que el empuje pulsional en acto propuesta por los estudios en adolescencia mencionados anteriormente. Bajo esto, la separación toma otras modalidades en donde emerge la noción de un objeto virtual que busca 


\section{ARTIGOS}

operar como limite ante la angustia y depresión adolescente (Disarbois, 2009).

Por esto, Marty (2010) conceptualiza la importancia de la separación de los padres también como un proceso que no es inicialmente facilitador de la autonomía, sino que también produce angustia para el adolescente y los padres. También destaca las referencias a Winnicott (1979) planteando cómo la muerte simbólica de los padres revela a los adolescentes su propia angustia y los confronta a esta: "Pero a veces el asesinato simbólico de los padres a los cuales se enfrentó el adolescente genera demasiada angustia y el riesgo de perder esos objetos de amor es demasiado grande como para oponerse a ellos. La dependencia de los objetos de los padres es a menudo parte del miedo a la pérdida y la angustia que tal temor despierta o genera" (Marty, 2010, p. 482).

Marty (2010) refiere que los procesos de separación descritos en la adolescencia, en tanto necesidad diferenciarse de los padres y llegar a un proceso de autonomía que le permita en lo concreto la inserción al mundo adulto, son sumamente importantes, pero también difíciles para el adolescente. Surgen en estos momentos angustias depresivas, frente a lo difícil que es la realización de esta separación, pero también necesaria sobre los objetos parentales, tanto a un nivel corporal como también identitario.

De este modo, esta separación implicaría no sólo abandonar las figuras parentales de la infancia, sino también, abandonar este espacio de transición y tramitación en donde se pueden mantener ambos posicionamientos, lo cual se torna complejo en el contexto actual en donde la flexibilidad e inmediatez priman en el lazo social.

\section{b) El problema de las identificaciones en la adolescencia}

Un modo de elaborar esta separación y pérdidas propuestas es el duelo, que bajo los postulados de Freud (1917[1915]/2013) implica la identificación con el objeto perdido. Así, para poder tolerar la pérdida del objeto de amor, primero el sujeto construye una forma originaria de lazo afectivo con este objeto, lo que se trataría de una relación preedípica marcada por la relación canibalística (o identificación primaria). Y segundo, a partir de la existencia de un substituto regresivo de una elección de objetal abandonada. Ya en ausencia de toda catexis con el objeto de amor, el sujeto puede identificarse a este en la medida en que tienen un elemento en común: por desplazamiento, la identificación se producirá sobre otro punto. 
De Kernier y Marty (2010) destacan el rol de la identificación en el proceso de elaboración adolescente. La adolescencia no es sólo un momento de la vida, sino un proceso psíquico verdadero estrechamente ligado a la novedad fundamental de la aparición de la pubertad, proceso que será parte de la elaboración adolescente. La problemática somática y psíquica de la adolescencia requiere que el niño se convierta para integrar esta nueva metamorfosis con un nuevo trabajo psíquico para acompañar los cambios, manteniendo cierta continuidad en el sentido del ser: "Los seres humanos también mudan, en el momento de la adolescencia, y sus plumas son plumas prestadas; se dice a menudo que el adolescente que comienza a perder sus antiguas identificaciones toma el aspecto de algo prestado" (Mannoni, 1984, pp. 26-27).

El registro identificatorio se utiliza en gran medida como el lazo en la cuestión de las relaciones con los objetos: "el autoinvestimiento - refuerzo narcisista - disputando el investimiento en el otro con el riesgo de sentirse perdido o vaciado de si mismo en las experiencias de amor" (De Kernier y Marty, 2010, p. 480). Por esto, la adolescencia puede comprenderse como un problema de identificaciones, entendida por Manonni (1984) como una capacidad lúdica, en donde el jugar con las identificaciones es parte del rol del analista en la clínica psicoanalítica con adolescentes.

Para Marty (2003), la fantasía de muerte del padre es una necesidad en la estructuración - citando a Winnicott - el resultado de esta confrontación mortal ofrece la perspectiva de la identificación de los adolescentes. Pero al mismo tiempo, el lugar del padre es un lugar imposible de ocupar, por lo cual su asesinato no es tanto una necesidad de la fantasía, sino más bien para el autor un desastre en su realización psíquica. Al mismo tiempo, esta fantasía produce angustia, la cual puede relacionarse con lo planteado por Elizabeth, en tanto su miedo a la soledad es relacionado con el miedo a ser "adulto", pensando la adultez como un quiebre absoluto con las figuras parentales, emergiendo angustia por la soledad y abandono.

Este asesinato simbólico es pensado como una metáfora por el autor respecto a la tarea del niño: "renunciar a la violencia de sus impulsos asesinos para ganar capacidad de pensar y crear" (p. 22). La creación de estos proyectos culturales puede lograrse a través del proceso de la identificación con la función parental, en tanto es en esta función en donde se crean proyectos culturales y se puede proyectar el mundo interno - que había sido mediado por las operaciones de interdicción y sostén - hacia el exterior, rompiendo la continuidad de una perspectiva intrapsíquica. 


\section{ARTIGOS}

\section{c) La identificación con la función parental}

La construcción identitaria del adolescente se nutre de la reivindicación de la diferencia de las generaciones, la cual no basta con ser reconocida. En este punto se marca la salida de la adolescencia, responder por sus acciones, por sus actos, y también convertirse en responsable de otro como de uno mismo, mediante la noción de una autoría de un proyecto cultural. Esta proyección cultural es lo que Marty (2009b) denomina como "la identificación con la función parental" (p. 25). El pasaje que conduce de la proyección del mundo interno hacia los objetos externos con el sentimiento de su responsabilidad en este acto psíquico. Este pasaje se torna dificultoso en algunos adolescentes, debido a los procesos psíquicos e intersubjetivos descritos que están relacionados.

La identificación con la función parental implica, a partir de la identificación con los padres, ir más allá de ellos para poder construir un proceso generacional, que finalmente remite a la pregunta edípica: la posición del sujeto frente a sus orígenes, como primer punto de referencia el cual es la base de las identificaciones futuras que el adolescente realizará, entre ellas un sobre todo la identificación con la función parental (Marty, 2003, p. 13).

La filiación es una deuda, ya que implica el reconocimiento del adolescente de su propia genealogía en el universo simbólico. El límite es lo que hace que los padres transmitan el reconocimiento de una deuda, pensado la transmisión del orden simbólico de un modo que establezca relaciones familiares (Marty, 2003). Así, el adolescente debe ir desde el fantasma parricida a la identificación con la función parental (Marty, 2007).

De este modo, las vías identificatorias que los padres ofrecen al adolescente permiten también comprender los deseos puestos en el hijo, así como también los anhelos sobre su crecimiento y vida. Tales identificaciones pueden dejar al hijo indiferenciado del adulto, sin posibilidad de salida en tanto de su historia, la cual se transforma en pura repetición de otra historia (Janin, 2013).

La identificación para el adolescente implica una búsqueda de distintos modelos, que, si bien superficialmente pueden describirse como una búsqueda de referentes fuera de la familia, más bien implica un paso inicial que se refiere a la identificación con la función parental, la cual permite el acceso a la transformación de su mundo interno al mundo exterior mediante manifestaciones como el arte, amistades, amor, etc., las cuales emergen en la clínica con adolescentes, como en el caso descrito. 
El proyecto identificatorio del adolescente implica una búsqueda de modelos, la cual se inicia con la oferta que el medio parental le realiza. La puesta en interrogación y resignificación de las significaciones identitarias heredadas facilita la transformación en los modos de ser investido e identificado, buscando su propio proyecto o, como refiere Marty (2007), haciendo generación y conquistando su lugar en el mundo de los adultos, dando paso a la idea de que el adolescente es un adulto en virtual (Marty, 2010).

\section{Conclusiones}

A través de la revisión realizada, se busca dar cuenta de cómo los procesos socioculturales inciden no sólo en los modos de construir la adolescencia, la transición hacia la adultez y la comprensión de la función parental, sino a su vez en los modos de sufrimiento presentes en esta transición, los cuales toman dimensiones particulares que ilustraron en la viñeta revisado.

Frente a esto, inicialmente destaca el rol de lo virtual como parte del conflicto generacional en el contexto actual. Se destaca cómo las modalidades de quiebre generacional y conflicto de la adolescencia no sólo se identifican como parte de conductas de rebelión, riesgo o puesta en acto del empuje pulsional, sino también a través de la soledad y el aislamiento con los padres, mediante la participación de lo virtual como nuevo lazo social que emerge como ajeno y disruptivo frente a la visión de los adultos. Es en este espacio virtual donde además emerge una nueva visión de lo íntimo en la relación con la otredad, exponiendo el acceso a lo íntimo como facilitado por lo virtual. Al mismo tiempo, lo virtual surge como un espacio transicional materilizado (Waserman, 2008) que se puede relacionar con un nuevo espacio que ofrecería elementos para que los adolescentes puedan elaborar su malestar, facilitando a su vez un quiebre generacional con sus padres, en tanto este espacio virtual opera fuera de la visión parental, con un lazo sociocultural virtual.

La adolescencia es un período que socialmente ha ido perdiendo claridad en cuanto a su finalización y límites, siendo relevante en este proceso la transmisión de la función parental. En las problemáticas clínicas en la transición hacia la adultez, se identifican dificultades en la transmisión de la función parental como proceso que posibilitaría el advenimiento subjetivo de la adultez mediante los procesos de duelo e identificación. De este modo, emergería no sólo un proceso de duelo en la adolescencia, vinculado al abandono de la niñez, sino también un proceso de duelo de la adolescencia, 


\section{ARTIGOS}

vinculado al abandono de este espacio temporal y psíquico que posibilita un estado de transición constante entre la niñez y la adultez. La diferenciación de ambos duelos contribuye a la comprensión de las dificultades en la adolescencia, y sobre todo en la adolescencia tardía, así como a los particulares procesos psíquicos relacionados en ambos momentos, buscando diferenciarse de la visión homogeneizante de la adolescencia.

La identificación con la función parental busca ir hacia el origen con el fin de construir un proyecto de vida hacia el futuro. Este proceso muestra la temporalidad psíquica particular e ilustradora del psiquismo que puede encontrarse en la adolescencia (Cornejo, 2015). Al mismo tiempo, da cuenta de la relevancia de incluir aspectos históricos, no sólo del adolescente, sino también de sus padres. La inclusión de estos aspectos posibilitaría la comprensión de la identificación con la función parental no sólo como un proceso de identificaciones, sino también de subjetivación, en tanto incluye elementos simbólicos e históricos en el proceso de transición hacia la adultez.

Bajo esto, se hace relevante proponer la inclusión de los padres, mediante entrevistas, no sólo en un inicio del proceso, sino también en el dispositivo psicoterapéutico desde su ejercicio parental en la adolescencia tardía. Si bien los casos de adolescencia tardía de manera normativa podrían incluirse desde la lógica de la adultez, la elaboración de la transición hacia la adultez se propone como un proceso psíquico y atemporal que no sigue necesariamente las lógicas de lo normativo.

La identificación con la función parental es fundamental en el proceso de transición hacia la adultez, en tanto pone en juego elementos históricos del adolescente y sus padres, como transmisores de esta función. Esto lleva a problematizar en torno a las ofertas identificatorias con que cuentan los adolescentes en su transición hacia la adultez en la actualidad, considerando el contexto sociocultural y cómo este ha ido definiendo la adultez y adolescencia. Establecer un diálogo entre estos elementos y los elementos históricos subjetivos que emergen desde el adolescente y también desde sus padres respecto a la transmisión de la función parental posibilitaría contribuir con nuevas miradas para el estudio de la transición hacia la adultez en el dispositivo clínico.

\section{Referencias}

Aberastury, A., \& Knobel, M. (1971). La adolescencia normal: un enfoque psicoanalítico. Buenos Aires, Argentina: Paidos. 
Bilbao, A., \& Jofré, D. (2017). Acerca de los mecanismos de deconstrucción del orden social instituyente durante el momento adolescente. Rev. latinoam. psicopatol. fundam., 20(3), 436-450. DOI: http://dx.doi.org/10.1590/14154714.2017v20n3p436.2.

Blos, P. (1976). La transición adolescente. Buenos Aires, Argentina: Amorrortu.

Cahn, R. (1997). L'adolescent dans la psychanalyse: L'aventure de la subjectivation. Paris, France: Presses Universitaires de France.

Cornejo, R. (2015). Temporalidad psíquica y subjetivación en la adolescencia. Rev. latinoam. psicopatol. fundam. 18(1), 62-73. DOI: http://dx.doi.org/10.1590/14154714.2015v18n1p62.5.

Dávila, O., \& Ghiardo, F. (2012). Transiciones a la vida adulta: Generaciones y cambio social en Chile. Última Década, 37, 69-83. DOI: http://dx.doi. org/10.4067/S0718-22362012000200004.

De Kernier, N., \& Marty, F. (2010). L'adolescente et la mort. Cliniques méditerranéennes, 81(1), 181-198. doi:10.3917/cm.081.0181.

Disarbois, B. (2009). L'addiction au virtual: une présence sans absence. Psychotropes, 15(1), 41-58. DOI : 10.3917/psyt.151.0041.

Dolto, F. (1973). El caso Dominique. Buenos Aires, Argentina: Siglo XXI Editores.

774 Dolto, F. (1988). La causa de los adolescentes. Barcelona, Madrid: Editorial Seix Barral.

Erikson, E. (1968). Identidad, juventud y crisis. Barcelona, Madrid: Editorial Taurus.

Feixa, C. (2006). Generación XX. Teorías sobre la juventud en la era contemporánea. Rev.latinoam.cienc.soc.niñez juv, 4(2), Manizales. Recuperado el 13 Jul. 2018 desde $<$ http://www.scielo.org.co/scielo.php?script=sci_arttext\&pid=S1692$715 \times 2006000200002>$.

Ferté-Marriaux, K. (2009). La construction de la parentalité à l'adolescence. Le Journal des psychologues, 269(6), 65-69. DOI: 10.3917/jdp.269.0065.

Freud, S. (1992). La novela familiar del neurótico. In Obras Completas (Vol. IX, pp. 217-220). Buenos Aires, Argentina: Amorrortu. (Trabalho original publicado em 1909).

Freud, S. (2013). Duelo y melancolía. In Obras Completas (Vol. XIV, pp. 241 -256). Buenos Aires, Argentina: Amorrortu. (Trabalho original publicado em 1917[1915]).

Gutton, P. (1993). Lo puberal. Buenos Aires, Argentina: Amorrortu.

Gutton, P. (2006). Parentalité. Adolescence, 55(1), 9-32. DOI:10.3917/ado.055.0009.

Horenstein, M. (2013). Con la navaja del padre. Adolescencia y cuestión del padre. Revista Uruguaya de Psicoanálisis, 117, 104-128. Recuperado el 3 Mayo 2018 desde $<$ http://www.apuruguay.org $>$. 


\section{ARTIGOS}

Janin, B. (2013). Intervenciones en la clínica psicoanalítica con niños. Buenos Aires, Argentina: Centro de Publicaciones Educativas y Material Didáctico.

Jeammet, P. (1995). La identidad y sus trastornos en la adolescencia. Revista de la Sociedad Española de Psiquiatría y Psicoterapia del Niño y del Adolescente, 19-20, 161-177.

Klein, A. (2014). Exploración de las ideas de Winnicott sobre la adolescencia y el conflicto de generaciones. Estudos de Psicología, 31(2), 169-178. DOI: http:// dx.doi.org/10.1590/0103-166X2014000200003.

Le Breton, D. (2012). La edad solitaria. Adolescencia y sufrimiento. Santiago, Chile: LOM Editores.

Mannoni, O. (1984). ¿Es analizable la adolescencia? In O. Mannoni, A. Deluz, B. Gibello, \& J. Hébrard. (ed.), La crisis de la Adolescencia. Barcelona, Madrid: Gedisa.

Marty, F. (2001). Les parents face au risque de la violence des enfants et des adolescents. Le Carnet PSY, 64(4), 25-33. DOI:10.3917/lcp.064.0025.

Marty, F. (2003). La parentalité: un nouveau concept pour quelles réalities?, la place du pére. Le Carnet PSY, 81(4), 27-33.

Marty, F. (2005). Hacia una tercera anamorfosis en la teoría de la sexualidad. In A. Birraux, M. Frioni, A. Ginés, P. Huerre, L. Kancyper, D. Lauru, I. Maggi, F. Marty, O. Ouvry, M. L. Pelento, F. Pommier, C. E. Prego, M. Ulriksen, \& M. Viñar, Adolescentes hoy en la frontera entre lo psíquico y lo social (pp. 14-21). Montevideo, Chile: Trilce.

Marty, F. (2007). Soutiens narcissiques à l'adolescence. Le Journal des psychologues, 245(2), pp. 22-27. DOI:10.3917/jdp.245.0022.

Marty, F. (2009a). La adolescencia como experiencia límite. In Bilbao, A., \& Morlans, I. Subjetivación, adolescencia, institución: psicopatología clínica y social (pp. 61-86). Chile, LOM Impresiones.

Marty, F. (2009b). ¿La psychothérapie psychanalytique d'adolescent existe-t-elle? Le Carnet PSY, 135(4), 22-29. DOI:10.3917/lcp.135.0022.

Marty, F. (2010a). Adolescence et monde virtual. Études, Tom. 413, 473-484.

Marty, F. (2010b). L'adolescente et la mort. Cliniques Méditerranées, 81, 181-198.

Marzana, D., Pérez-Acosta, A., Marta, E., \& González, M. (2010). La transición a la edad adulta en Colombia: una lectura relacional. Avances en Psicología Latinoamericana, 28(1), 99-112. DOI: http://dx.doi.org/10.22458/rpys.v14i2.1629.

Piccini, Barrionuevo \& Vega. (2007). Escritos psicoanalíticos sobre adolescencia. Buenos Aires, Argentina: Eudeba.

Rassial, J. (1999). El pasaje adolescente. De la familia al vínculo social. Barcelona, Espanha: Ediciones del Serbal. 
Richard, F. (2009). Adolescencia y nuevo malestar en la cultura. In Bilbao, A., \& Morlans, I. Subjetivización, adolescencia, institución: psicopatología clínica y social. Santiago, Chile: LOM Impresiones.

Rother de Hornstein, M. (2006). Adolescencias: trayectorias turbulentas. Buenos Aires, Argentina: Paidos.

Untoiglich, G. (2009). Patologías actuales en la infancia. In L. Wettengell, G. Untoiglich, \& G. Szyber, Patologías actuales en la infancia. Bordes y desbordes en clínica y educación (pp 13-34). Buenos Aires, Argentina: Noveduc.

Valdés, X. (2007). Notas sobre la metamorfosis de la familia en Chile. Reunión de especialistas Futuro de las familias y desafios para las politicas públicas CEPAL, UNFPA. Recuperado el 2 Mayo 2014 desde: <http://www.eclac.org/dds/noticias/ paginas/9/30289/Resumen.XimenaValdes.pdf $>$.

Waserman, M. (2008). Aproximaciones psicoanaliticas al juego y al aprendizaje: ensayos y errores. Buenos Aires, Argentina: Centro de Publicaciones Educativas y Material Didáctico.

Wettengell, L. (2009). Trazando surcos: el trabajo de la parentalidad. In L. Wettengell, G. Untoiglich, \& G. Szyber, Patologías actuales en la infancia. Bordes y desbordes en clínica y educación (pp. 35-52). Buenos Aires, Argentina: Noveduc.

Winnicott, D. (1964). Deprivación y Delincuencia. Buenos Aires, Argentina: Paidos. Winnicott, D. (1979). Realidad y Juego. Barcelona, Madrid: Gedisa.

\section{Resumos}

(Análise psicanalítica sobre os problemas na identificação com a função parental na adolescência: a transição para a vida adulta como espaço de transformação)

Por meio de uma vinheta clínica, a proposta é analisar como, durante a adolescência, ocorre um processo de identificação e transmissão da função parental na relação entre o adolescente e seus pais, o que permitiria pensar nos avatares dos processos de transição para a vida adulta. Discute-se como a inclusão desses processos na clínica com adolescentes poderia enriquecer as análises com perspectivas históricas e intersubjetivas que possibilitariam refletir sobre o papel dos pais na clínica com adolescentes, sobre o luto com relação à adolescência e sobre os problemas atuais na transição para a vida adulta.

Palavras-chave: Função parental, adolescência, vida adulta, identificação 


\section{ARTIGOS}

(Psychoanalytic analysis about identification problems with the parental function in adolescence: transition toward adulthood as a transformative space)

A clinic vignette is used to analyze how in adolescence the process of identification and transmission of parental function is realized in the relation between adolescents and their parents. This analysis could allow us to think about the processes of transition toward adulthood. The inclusion of these processes in the clinic with adolescents could enrich the analyses with intersubjective and historical perspectives, which would allow to reflecting on the roles of parents in the clinic with adolescents, the grief in relation to the adolescence, and the current problems in the transition toward adulthood.

Key words: Parental function, adolescence, adulthood, identification

(Analyse psychanalytique sur les problématiques concernant l'identification à la fonction parentale durant l'adolescence: la transition vers l'âge adulte comme espace de transformation)

En utilisant une vignette clinique à titre d'illustration, ce travail vise à analyser comment, à l'adolescence, a lieu un processus d'identification et de transmission de la fonction parentale dans la relation entre l'adolescent et ses parents, ce qui pourrait permettre de penser aux vicissitudes des processus de transition vers l'âge adulte. Une discussion est menée pour déterminer comment l'inclusion de ces processus à la clinique avec des adolescents pourrait approfondir les analyses en adoptant des perspectives historiques et intersubjectives, ce qui permettrait de réfléchir au rôle des parents à la clinique avec des adolescents, au deuil de sa propre adolescence et aux problématiques actuelles lors de la transition vers l'âge adulte pour des adolescents.

Mots clés: Fonction parentale, adolescence, âge adulte, identification

(Psychoanalytische Analyse von Problemen bei der Identifikation mit der elterlichen Funktion in der Adoleszenz: Der Übergang ins Erwachsenenalter als Raum für Transformation)

Anhand einer klinischen Vignette analysiert dieser Artikel, wie in der Adoleszenz der Prozess der Identifikation und Übertragung der elterlichen Funktion in der Beziehung zwischen Jugendlichen und ihren Eltern stattfindet, was uns erlaubt, über die Avatare der Transitionsprozesse ins Erwachsenenalter nachzudenken. Es wird diskutiert, wie die Einbeziehung dieser Prozesse in die Klinik von Jugendlichen die Analysen mit historischen und intersubjektiven Perspektiven bereichern könnte, was außerdem ermöglichen würden, die Rolle der Eltern in der Klinik von Jugendlichen zu überdenken, sowie die Trauer in Bezug auf die eigene Adoleszenz und die aktuellen Probleme von Jugendlichen beim Übergang ins Erwachsenenalter.

Schlüsselwörter: Elterliche Funktion, Adoleszenz, Erwachsenenalter, Identifikation 
Citação/Citation: Olguin, D. F. (2018, dezembro). Análisis psicoanalítico sobre las problemáticas en la identificación con la función parental en la adolescencia: La transición hacia la adultez como un espacio de transformación. Revista Latinoamericana de Psicopatologia Fundamental, 21(4), 761-778. http://dx.doi.org/10.1590/1415-4714.2018v21n4p761.5.

Editores do artigo/Editors: Profa. Dra. Ana Maria G. R. Oda e Profa. Dra. Sonia Leite.

Recebido/Received: 4.4.2018/ 4.4.2018 Aceito/Accepted: 15.7.2018 / 7.15.2018

Copyright: (C) 2009 Associação Universitária de Pesquisa em Psicopatologia Fundamental/ University Association for Research in Fundamental Psychopathology. Este é um artigo de livre acesso, que permite uso irrestrito, distribuição e reprodução em qualquer meio, desde que o autor e a fonte sejam citados / This is an open-access article, which permits unrestricted use, distribution, and reproduction in any medium, provided the original authors and sources are credited.

Financiamento/Funding: Este trabalho não recebeu financiamento / This work received no funding.

Conflito de interesses/Conflict of interest: A autora declara que não há conflito de interesses / The author declares that there is no conflict of interest.

\section{Daniela Fernández Olguín}

Psicóloga; Magíster en Clínica Psicoanalítica con Niños y Jóvenes; Docente en Universidad Viña del Mar (Viña del Mar,Chile).

Los Fresnos 91, 2520000, Viña del Mar, Chile. daniela.paz.f@gmail.com

This is an open-access article, which permits unrestricted use, distribution, and reproduction in any medium for non-commercial purposes provided the original authors and sources are credited. 